\title{
Changes in circulating endothelial progenitor cells predict responses of multiple myeloma patients to treatment with bortezomib and dexamethasone
}

\author{
L. Wang ${ }^{1}$, F. Du ${ }^{2}$, H.M. Zhang ${ }^{1}$, W.J. Zhang ${ }^{1}$ and H.X. Wang ${ }^{1}$ \\ ${ }^{1}$ Department of Hematology, The Central Hospital of Wuhan, Tongji Medical College, \\ Huazhong University of Science and Technology, Wuhan, China \\ ${ }^{2}$ Department of Gastroenterology, Union Hospital, Tongji Medical College, \\ Huazhong University of Science and Technology, Wuhan, China
}

\begin{abstract}
Four cycles of chemotherapy are required to assess responses of multiple myeloma (MM) patients. We investigated whether circulating endothelial progenitor cells (cEPCs) could be a biomarker for predicting patient response in the first cycle of chemotherapy with bortezomib and dexamethasone, so patients might avoid ineffective and costly treatments and reduce exposure to unwanted side effects. We measured cEPCs and stromal cell-derived factor- $1 \alpha$ (SDF-1 $\alpha$ ) in 46 MM patients in the first cycle of treatment with bortezomib and dexamethasone, and investigated clinical relevance based on patient response after four 21-day cycles. The mononuclear cell fraction was analyzed for cEPC by FACS analysis, and SDF-1 $\alpha$ was analyzed by ELISA. The study population was divided into 3 groups according to the response to chemotherapy: good responders ( $n=16$ ), common responders $(n=12)$, and non-responders $(n=18)$. There were no significant differences among these groups at baseline day $1(\mathrm{P}>0.05)$. cEPC levels decreased slightly at day $21(8.2 \pm 3.3 \mathrm{cEPCs} / \mu \mathrm{L})$ vs day $1(8.4 \pm 2.9 \mathrm{cEPCs} / \mu \mathrm{L}) \mathrm{in} \mathrm{good}$ responders $(P>0.05)$. In contrast, cEPC levels increased significantly in the other two groups $(P<0.05)$. SDF-1 $\alpha$ changes were closely related to changes in cEPCs. These findings indicate that change in cEPCs at day 21 in the first cycle might be considered a noninvasive biomarker for predicting a later response, and extent of change could help decide whether to continue this costly chemotherapy. cEPCs and the SDF-1 $\alpha /$ CXCR4 axis are potential therapeutic targets for improved response and outcomes in MM patients.
\end{abstract}

Key words: Chemotherapy; Circulating endothelial progenitor cells; Multiple myeloma

\section{Introduction}

Bortezomib plus dexamethasone has been recommended as a primary therapy for multiple myeloma (MM) stem cell transplant and non-transplant candidates. After four 3-week cycles of the regimen, the rate of complete response (CR)/near CR was $14.8 \%$, the rate of achieving at least a very good partial remission (VGPR) was $37.7 \%$, and the overall response rate was $78.5 \%(1,2)$. However, four cycles are needed to assess a response to this valuable but costly regimen. Therefore, it would be particularly valuable in developing countries to identify a biomarker that could predict the possibility of a response in the later phase of treatment. In recent years, circulating endothelial progenitor cells (cEPCs) have been documented to change during anti-angiogenic therapy, and cEPC levels were thought to be useful as a predictive marker for monitoring chemotherapy, particularly with anti-angiogenic therapies for some cancers, such as breast cancer, non-small-cell lung cancer, prostate cancer, and MM (3-6). cEPC numbers were significantly increased in MM patients and correlated with serum $\beta_{2}$-microglobulin levels (7). After a median follow-up of 4 months, the responders demonstrated a significant decrease in CEPC numbers compared to their pretreatment values (5). In addition to cEPCs, angiopoietins and a novel panel of proteins were also considered as potential predictive biomarkers for treating MM (8,9). However, these biomarkers were not studied in treatments with bortezomib. In particular, some thalidomide non-responders successfully achieved CR/VGPR using

Correspondence: Hong-xiang Wang: <397959379@qq.com>. 
bortezomib; thus, it is debatable whether these potential biomarkers are still efficacious. The changes in cEPC levels after 7 days of chemotherapy correlated with the tumor volumes after three cycles of chemotherapy and predicted progression-free survival (PFS) or overall survival (OS), regardless of the chemotherapy regimens used with some tumors. Until now, MM biomarkers have been measured prior to therapy or after four cycles of chemotherapy. However, the values in the early phase of the first cycle of chemotherapy may be more predictive.

In this study, we specifically focused on whether changes in cEPC levels in the early phase of the first cycle of chemotherapy could predict the response of newly diagnosed MM patients to a regimen of bortezomib and dexamethasone. Because stromal cell-derived factor- $1 \alpha$ (SDF-1 $\alpha$ ) plays a key role in both the release and homing processes of cEPCs, we attempted to determine whether it also correlated with the cEPC changes observed during chemotherapy. This study could potentially assist physicians in choosing a bortezomib-based treatment, thereby avoiding other ineffective and costly treatments; it would be particularly valuable for patients in developing countries.

\section{Material and Methods}

\section{Study population}

A total of 50 newly diagnosed MM patients were registered at our hospital between May 2009 and November 2014. In the end, 46 patients were available after a 4-month period to assess the response. They underwent four 21-day cycles of bortezomib (Velcade, Millennium Pharmaceuticals, USA) plus dexamethasone $(\mathrm{n}=46)$ : bortezomib $\left(1.3 \mathrm{mg} / \mathrm{m}^{2}\right)$ intravenous bolus on days $1,4,8$, and 11 , and dexamethasone $(20 \mathrm{mg})$ on days $1,2,4,5,8,9,11$, and 12 . For each patient, a baseline peripheral blood (PB) sample was drawn prior to therapy, and then PB samples were collected on days 7 , 14, and 21. Routine laboratory data including serum concentrations of $\beta_{2}$-microglobulin, M-protein, albumin, and International Staging System (ISS) stages were obtained from medical records. Thirty-five patients had adverse events such as constipation, peripheral neuropathy, thrombocytopenia, leucopenia, and secondary high glucose levels. The study was approved by the Ethics Committee of the Central Hospital of Wuhan. All patients provided signed informed consent prior to the study.

\section{Inclusion and exclusion criteria}

The inclusion criteria included age (18-65 years) and a diagnosis of $\mathrm{MM}$ with the International Myeloma Working Group diagnostic criteria. The exclusion criteria were a history of inflammatory or infectious cardiovascular or autoimmune disease, use of steroids prior to the start of therapy, use of erythropoiesis-stimulating agents or granulocyte-colony stimulating factor (G-CSF), and infectious diseases such as herpes zoster. Among the 50 patients who participated in the study, 2 patients did not complete four cycles of chemotherapy and 2 patients who suffered from herpes zoster were excluded.

\section{Enumeration of cEPCs by flow cytometry}

Using EDTA as an anticoagulant, peripheral venous blood was collected from the MM patients at four time points during the first cycle of chemotherapy. The first day of the first cycle of chemotherapy was considered to be day 1. Samples were collected in the mornings on days 1 , 7,14 , and 21 (the day prior to the start of the second cycle of chemotherapy). cEPCs were measured twice at each time point, and the average was used for evaluation. PB mononuclear cells from anticoagulated blood were separated using Ficoll (BD Biosciences, USA). The tubes were centrifuged at room temperature for $20 \mathrm{~min}$ at $1800 \mathrm{~g}$ and then washed twice with PBS. Mononuclear cells $\left(5 \times 10^{6}\right)$ were labeled with preconjugated mouse anti-human monoclonal antibodies: CD34 conjugated to allophycocyanin, CD309 (VEGF receptor-2 [VEGFR2]/kinase insert domain-containing receptor [KDR]) to fluorescein isothiocyanate (BD Biosciences, USA), and CD45 to peridinin chlorophyll protein-cyanine for $20 \mathrm{~min}$ at room temperature. The mixture was then incubated at $37^{\circ} \mathrm{C}$ for $30 \mathrm{~min}$ in the dark, washed with PBS, and then suspended in $1 \%$ paraformaldehyde and maintained at $4^{\circ} \mathrm{C}$ prior to analysis. CEPCs were evaluated by four-color flow cytometry (BD FACSAria II) and were identified as CD45-/dim cells with coexpression of CD34 and CD309. Although there are many methods (using different antibodies) for identifying cEPCs, CD34 and CD309 double-positive cells were considered to be cEPCs, an approach that is supported by many biological and methodological studies (10-12). The number of cells per milliliter of blood was calculated and compared to the mononuclear cell count of the original sample.

\section{Quantification of serum levels of SDF- $1 \alpha$ by ELISA}

All PB samples were collected in potassium-EDTA or serum tubes. SDF- $1 \alpha$ levels were determined in PB samples using a commercially available SDF- $1 \alpha$ ELISA kit (BD Biosciences) according to the manufacturer's instructions.

\section{Assessment of response}

Treatment response was assessed according to the international uniform response criteria for multiple myeloma (13). Briefly, complete response (CR) was defined as an undetectable level of serum M-protein and $\leqslant 5 \%$ bone marrow plasma cells (BMPCs), VGPR was defined as a reduction in serum $M$-protein by at least $90 \%$ of the initial value, and partial response (PR) was defined as a reduction in M-protein by approximately $50-90 \%$. Patients with a reduction of $\leqslant 50 \% \mathrm{M}$-protein were defined as having stable disease (SD), and those with either an 
increase in M-protein of $\geqslant 25 \%$ from baseline or an increase in BMPCs of $\geqslant 10 \%$ were defined as having progressive disease (PD). Patients who achieved PR or better response were considered responders. Patients who achieved SD, PD, or a worse effect were considered non-responders.

\section{Statistical analysis}

Continuous variables are reported as means $\pm S D$. Categorical variables are reported as number or percentage. Statistical comparisons were performed using the independent-samples and paired-samples $t$-test. Paired-samples $t$-test was used to estimate statistically significant differences in cEPC numbers between patients in different groups. The correlation between CEPC numbers and SDF- $1 \alpha$ was evaluated using a Pearson correlation test when the data were normally distributed. All results were analyzed using SPSS 15.0 (SPSS, Inc., USA), and $\mathrm{P}<0.05$ (two-sided) was considered to be statistically significant.

\section{Results}

\section{Patient characteristics}

Fifty MM patients were enrolled in the study; however, 4 patients did not complete the four chemotherapy cycles and, therefore, 46 patients were available for the analysis of changes in cEPCs and SDF- $1 \alpha$ after chemotherapy. Patients were assigned to one of two groups, according to their responses. Eighteen patients fulfilled the criteria of $\mathrm{PR}$ or better responses and were considered to be the responders, while the remaining 28 patients who achieved $\mathrm{SD}, \mathrm{PD}$, or more serious effects were considered to be the non-responders. There were no significant differences between the two groups in terms of factors such as age, gender, M-protein, $\beta_{2}$-microglobulin, serum albumin, and ISS stage. The patient demographics and clinical staging are shown in Table 1.
cEPC changes during the first cycle of chemotherapy

Overall, an increase was observed in cEPCs after the first cycle of chemotherapy. After 7 and 21 days, the increase in cEPCs was substantially greater than that at the beginning, and they were consistently present. At day 7 , cEPC levels increased to $141 \%(95 \% \mathrm{Cl}=128-154 \%$, $\mathrm{P}<0.01$ ). At day 14, cEPC levels further increased to $231 \%(95 \% \mathrm{Cl}=210-253 \%, \mathrm{P}<0.01)$. Then, at day 21 , cEPC levels decreased to $188 \%(95 \% \mathrm{Cl}=163-213 \%$, $\mathrm{P}<0.01$ ), as shown in Figures 1 and 2.

\section{SDF-1 $\alpha$ changes in the first cycle of chemotherapy}

As shown in Figure 3, at baseline, SDF- $1 \alpha$ was $3530 \mathrm{pg} / \mathrm{mL} \quad(95 \% \mathrm{Cl}=3292-3768 \mathrm{pg} / \mathrm{mL})$. After 7 and 14 days, SDF- $1 \alpha$ levels steadily increased to $4253 \mathrm{pg} / \mathrm{mL}$ $(95 \% \mathrm{Cl}=3920-4586 \mathrm{pg} / \mathrm{mL}, \quad \mathrm{P}<0.01)$ and $4819 \mathrm{pg} / \mathrm{mL}$ $(95 \% \mathrm{Cl}=4491-5148 \mathrm{pg} / \mathrm{mL}, \mathrm{P}<0.01)$. At day $21, \mathrm{SDF}-1 \alpha$ levels decreased to $4112 \mathrm{pg} / \mathrm{mL}(95 \% \mathrm{Cl}=3811-4414 \mathrm{pg} / \mathrm{mL}$, $\mathrm{P}<0.01)$ but remained higher than the baseline level. There was a significant positive correlation between CEPC and SDF- $1 \alpha$ at 7 days (Pearson $r=0.48, P=0.02$ ), 14 days (Pearson $r=0.53, P=0.01$ ), and 21 days (Pearson $r=0.482$, $\mathrm{P}=0.02$ ) after chemotherapy.

\section{Changes in CEPC levels and response}

After four cycles of chemotherapy, CR was achieved in $5 / 46$ patients $(10.9 \%)$, VGPR in $11 / 46$ patients $(23.9 \%)$, and $\mathrm{PR}$ in $12 / 46$ patients $(26.1 \%)$; and $18 / 46$ patients $(34.8 \%)$ had SD or PD. The study population was divided into 3 groups according to the response to chemotherapy: good responders (CR + VGPR, $n=16)$, common responders $(P R, n=12)$, and non-responders (SD + PD, $n=18)$. There were no significant differences among these 3 groups at the baseline day 1 (chi-square=2.169, $\mathrm{P}=0.338$ ), and $c E P C$ numbers in the 3 groups were (means $\pm \mathrm{SE}$ ) $8.4 \pm 2.9,9.8 \pm 4.5$, and $10.3 \pm 4.0 \mathrm{cEPCs} / \mu \mathrm{L}$, respectively, as shown in Figure 4.

Table 1. Patient characteristics.

\begin{tabular}{lccc}
\hline Characteristic & MM patients $(\mathrm{n}=46)$ & Responders $(\mathrm{n}=18)$ & Non-responders $(\mathrm{n}=\mathbf{2 8})$ \\
\hline Age (years) & $54.1 \pm 4.8$ & $52.2 \pm 3.8$ & $55.2 \pm 2.2$ \\
Male/female & $29 / 17$ & $10 / 8$ & $19 / 9$ \\
M-protein (g/L) & $33.5 \pm 6.2$ & $32.0 \pm 5.8$ & $34.1 \pm 7.0$ \\
$\beta_{2}$-MG (mg/L) & $4.9 \pm 0.7$ & $4.6 \pm 0.5$ & $5.1 \pm 0.8$ \\
Serum albumin (g/L) & $38.2 \pm 4.1$ & $38.6 \pm 3.9$ & $35.8 \pm 5.2$ \\
ISS stage & 11 & 7 & 4 \\
I & 20 & 8 & 12 \\
II & 15 & 5 & 7 \\
III & & & \\
\hline
\end{tabular}

Data are reported as means $\pm S D$ or number of continuous and categorical variables. $\beta_{2}-\mathrm{MG}$ : $\beta_{2}$-microglobulin; ISS: International Staging System. There were no statistical differences between groups $(P>0.05$, independent samples $t$-test for continuous variables and chi square test for categorical variables). 

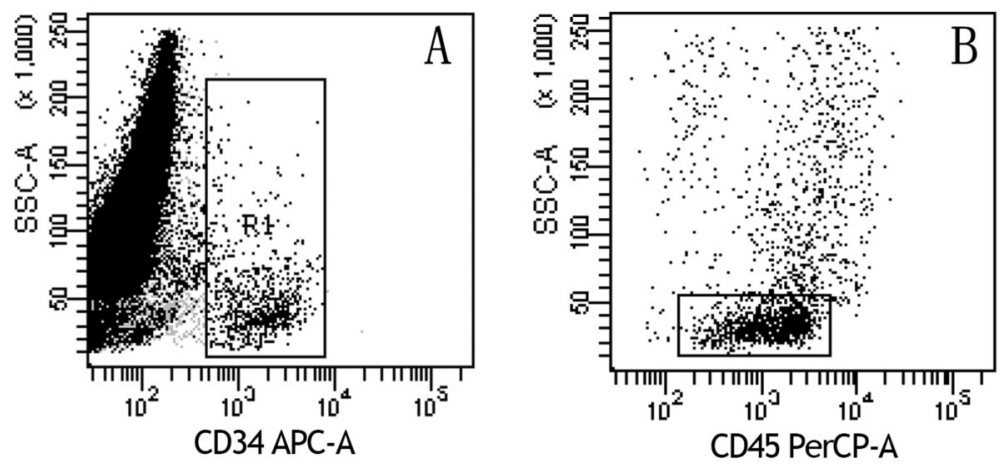

Figure 1. Representative flow cytometric analysis plots showing sequential gating strategy used to enumerate circulating endothelial progenitor cells (cEPCs): CD34 + and CD45-/dim cells were gated to exclude hematopoietic cells expressing CD45 antigen $(A, B)$. $C$, Negative control, and $D$, cells co-expressing CD34 and CD309 were designated as cEPCs.
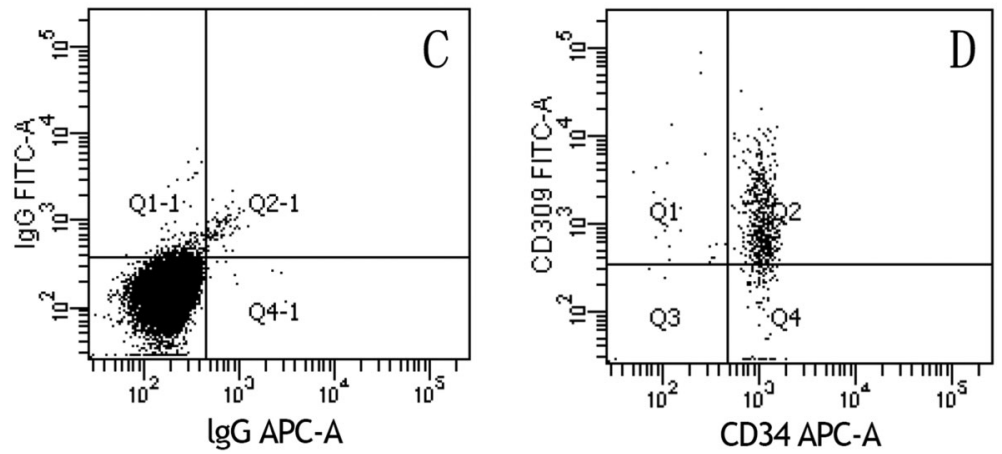

cEPC levels decreased minimally at day $21(8.2 \pm 3.3)$ compared with day $1(8.4 \pm 2.9)$ in the subgroup of good responders, but this was not statistically significant $(P=0.775)$. In contrast, cEPC levels in the other two groups increased significantly. Within the PR group, cEPC levels increased to $16.2 \pm 5.1$ from $9.8 \pm 4.5(P=0.002)$, while cEPC levels increased progressively to $33.4 \pm 9.8$ from $10.3 \pm 4.0 \quad(P<0.001)$ within the non-responders group.

\section{Discussion}

Bone marrow angiogenesis played an important role in the pathogenesis and progression of MM (14). It is well documented that the anti-myeloma effect of bortezomib partly occurs through a reduction of angiogenesis. Patients who responded well were most likely to have a significant decrease in microvessel density, whereas patients with stable or increased microvessel density experienced a relapse (15). The fact that angiogenin levels decreased following bortezomib treatment also suggested an anti-angiogenic mechanism. In vitro, bortezomib also produced an anti-angiogenic effect in MM patient-derived endothelial cells. Dexamethasone could functionally attenuate endothelial responses to VEGF, which plays an important role in the angiogenesis of $\mathrm{MM}$ (16). cEPCs are increased in MM patients and correlated with angiogenesis (7). In this study, we confirmed that the extent of change for CEPC numbers from day 1 to day 21 during the first cycle of treatment could predict the efficacy after four cycles. The smaller the change the more likely it is to achieve a better clinical effect. These results suggest that if an MM patient's angiogenesis was not inhibited effectively in the first cycle of chemotherapy with this

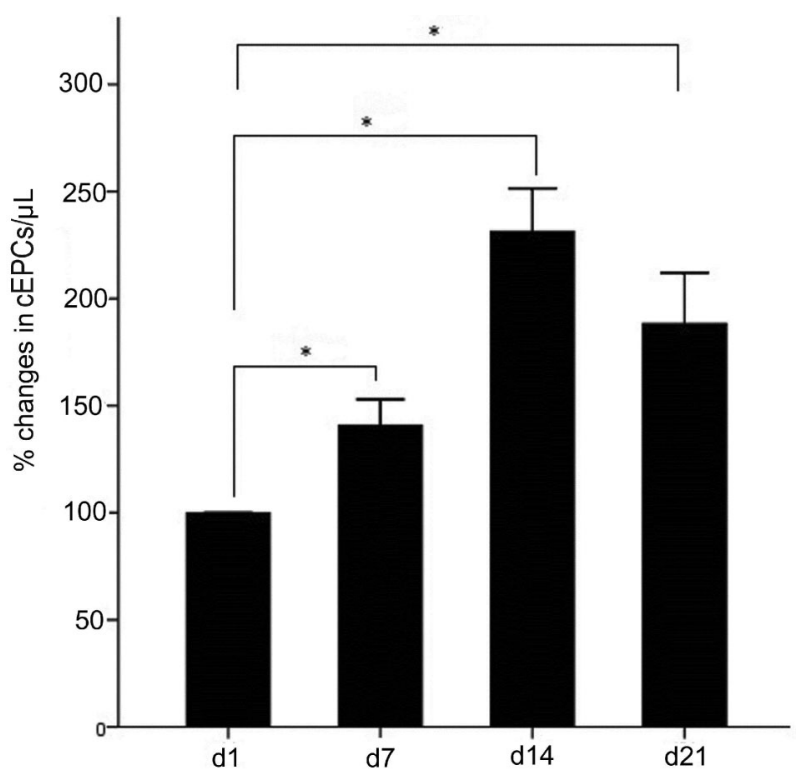

Figure 2. Kinetics of circulating endothelial progenitor cells (cEPCs) in the first cycle of chemotherapy. d: day. ${ }^{*} \mathrm{P}<0.01$ (paired-samples $t$-test). 


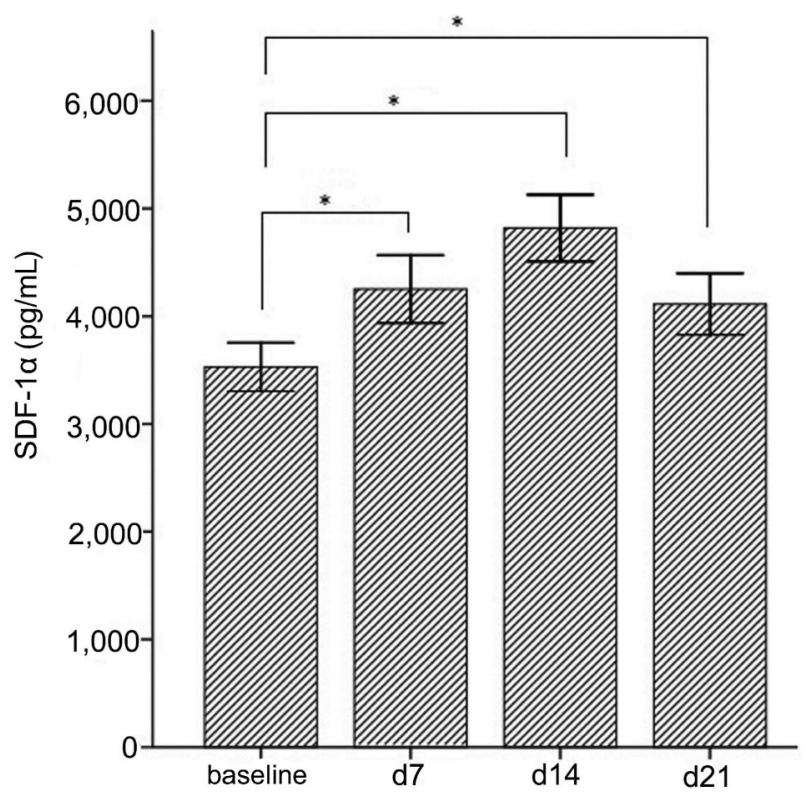

Figure 3. Kinetics of stromal cell-derived factor (SDF)- $1 \alpha$ changes in the first cycle of chemotherapy. d: day. ${ }^{*} \mathrm{P}<0.01$ (paired-samples $t$-test).

regimen, then the patient might have a poor response, even after four cycles.

In addition to targeting myeloma plasma cells, antimyeloma drugs, such as thalidomide, lenalidomide, bortezomib, and dexamethasone, also exert direct or indirect anti-angiogenic effects. Many angiopoietins, such as basic fibroblast growth factor (bFGF) and angiopoietin-2 (Ang-2), were found to correlate with VEGF and cEPCs in MM patients; therefore, the addition of an anti-angiopoietin agent may improve the efficacy of anti-myeloma therapy (8). SDF$1 \alpha$ is known for its key role in both the release and homing of cEPCs (17). It has been reported that SDF- $1 \alpha$ is increased in $\mathrm{MM}$ and correlated with the load and angiogenesis of myeloma plasma cells $(18,19)$. The SDF-1 $\alpha /$ CXCR4 axis has been shown to play an important role in neovascularization, metastasis, and chemotherapy resistance in MM. Here, we found SDF- $1 \alpha$ was also correlated with levels of cEPCs in MM during the first cycle of chemotherapy. Conceptually, these findings point to an array of new therapeutic strategies by combining chemotherapy with agents capable of inhibiting the release of progenitor cells, such as SDF-1 $\alpha /$ CXCR4 antagonists, which may enhance the therapeutic potential of conventional chemotherapy (2023). Both dexamethasone and thalidomide played an indirect role in targeting the SDF-1 $\alpha / C X C R 4$ axis $(24,25)$. AMD3100, a small-molecule CXCR4 antagonist, has been used to collect hematopoietic stem cells for transplantation in MM patients (26). In addition, it could enhance the tumor reduction induced by bortezomib (27).

This study has several limitations. First, we lacked a long-term follow-up; therefore, we did not have the patients' PFS and OS data for further in-depth analysis. Second, because of the small sample size, we could not provide a definite threshold for the cEPC changes necessary to predict the response.

In conclusion, this study has shown that chemotherapy evokes a host response that is composed of the release of cEPCs and SDF- $1 \alpha$. The extent of this release during the first cycle of chemotherapy correlates with the response four cycles later. cEPCs might be an early predictor of therapy response. Furthermore, SDF-1 $1 \alpha$ might be the key

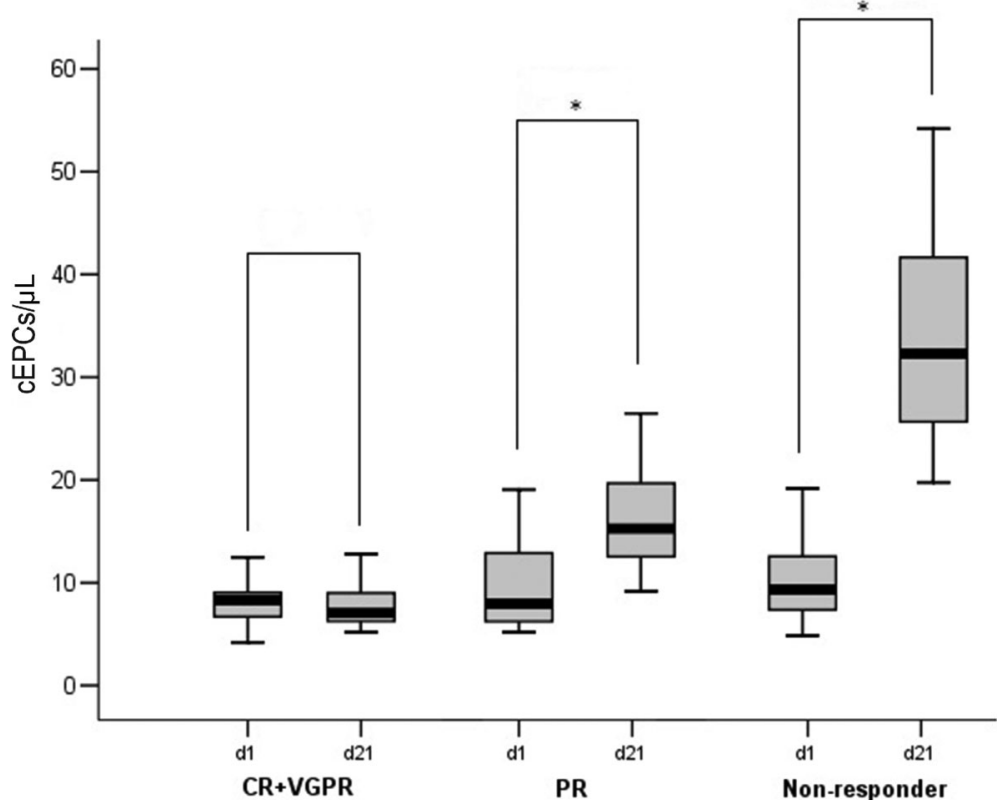

Figure 4. Box-plot representation of the circulating endothelial progenitor cells (cEPCs) levels at days 1 and 21. Box-plots show median (middle line), interquartile range (box), 25-75th percentile (whiskers). CR: complete response; VGPR: very good partial remission; PR: partial response; d: day. The paired-samples $t$-test was used for statistical analyses. 
reason for cEPC changes, and targeting SDF-1 $\alpha / C X C R 4$ axis therapy or combining it with conventional chemotherapy could improve the responses and outcomes of MM patients.

\section{References}

1. Harousseau JL, Attal M, Leleu X, Troncy J, Pegourie B, Stoppa AM, et al. Bortezomib plus dexamethasone as induction treatment prior to autologous stem cell transplantation in patients with newly diagnosed multiple myeloma: results of an IFM phase II study. Haematologica 2006; 91: 1498-1505.

2. Rajkumar SV. Multiple myeloma: 2012 update on diagnosis, risk-stratification, and management. Am J Hematol 2012; 87: 78-88.

3. Roodhart JM, Langenberg MH, Vermaat JS, Lolkema MP, Baars A, Giles $\mathrm{RH}$, et al. Late release of circulating endothelial cells and endothelial progenitor cells after chemotherapy predicts response and survival in cancer patients. Neoplasia 2010; 12: 87-94, doi: 10.1593/neo. 91460 .

4. Sakamori $\mathrm{Y}$, Masago K, Ohmori K, Togashi $\mathrm{Y}$, Nagai H, Okuda $\mathrm{C}$, et al. Increase in circulating endothelial progenitor cells predicts response in patients with advanced nonsmall-cell lung cancer. Cancer Sci 2012; 103: 1065-1070, doi: 10.1111/cas.2012.103.issue-6.

5. Bhaskar A, Gupta R, Kumar L, Sharma A, Sharma MC, Kalaivani $\mathrm{M}$, et al. Circulating endothelial progenitor cells as potential prognostic biomarker in multiple myeloma. Leuk Lymphoma 2012; 53: 635-640, doi: 10.3109/10428194. 2011.628880.

6. Li H, Raia V, Bertolini F, Price DK, Figg WD. Circulating endothelial cells as a therapeutic marker for thalidomide in combined therapy with chemotherapy drugs in a human prostate cancer model. BJU Int 2008; 101: 884-888, doi: 10.1111/bju.2008.101.issue-7.

7. Zhang $\mathrm{H}$, Vakil V, Braunstein M, Smith EL, Maroney J, Chen $\mathrm{L}$, et al. Circulating endothelial progenitor cells in multiple myeloma: implications and significance. Blood 2005; 105: 3286-3294, doi: 10.1182/blood-2004-06-2101.

8. Bhaskar A, Gupta R, Vishnubhatla S, Kumar L, Sharma A, Sharma MC, et al. Angiopoietins as biomarker of disease activity and response to therapy in multiple myeloma. Leuk Lymphoma 2013; 54: 1473-1478, doi: 10.3109/10428194. 2012.745523.

9. Rajpal R, Dowling P, Meiller J, Clarke C, Murphy WG, O'Connor R, et al. A novel panel of protein biomarkers for predicting response to thalidomide-based therapy in newly diagnosed multiple myeloma patients. Proteomics 2011; 11: 1391-1402, doi: 10.1002/pmic.v11.8.

10. Fadini GP, Avogaro A. Cell-based methods for ex vivo evaluation of human endothelial biology. Cardiovasc Res 2010; 87: 12-21, doi: 10.1093/cvr/cvq119.

11. Masouleh BK, Baraniskin A, Schmiegel W, Schroers R. Quantification of circulating endothelial progenitor cells in human peripheral blood: establishing a reliable flow cytometry protocol. J Immunol Methods 2010; 357: 38-42, doi: 10.1016/j.jim.2010.03.015.

12. Van Craenenbroeck EM, Van Craenenbroeck $A H$, van lerssel S, Bruyndonckx L, Hoymans VY, Vrints CJ, et al.

\section{Acknowledgments}

This research was supported by the Wuhan Health and Family Planning Commission in 2012 (\#WX12A04).

Quantification of circulating CD34+/KDR +/CD45dim endothelial progenitor cells: analytical considerations. Int J Cardiol 2013; 167: 1688-1695.

13. Durie BG, Harousseau JL, Miguel JS, Blade J, Barlogie B, Anderson $\mathrm{K}$, et al. International uniform response criteria for multiple myeloma. Leukemia 2006; 20: 1467-1473, doi: 10.1038/ sj.leu.2404284.

14. Ria R, Reale A, De Luisi A, Ferrucci A, Moschetta M, Vacca A. Bone marrow angiogenesis and progression in multiple myeloma. Am J Blood Res 2011; 1: 76-89.

15. Roccaro AM, Hideshima T, Raje N, Kumar S, Ishitsuka K, Yasui $\mathrm{H}$, et al. Bortezomib mediates antiangiogenesis in multiple myeloma via direct and indirect effects on endothelial cells. Cancer Res 2006; 66: 184-191, doi: 10.1158/00085472.CAN-05-1195.

16. Igarashi J, Hashimoto T, Shoji K, Yoneda K, Tsukamoto I, Moriue $\mathrm{T}$, et al. Dexamethasone induces caveolin-1 in vascular endothelial cells: implications for attenuated responses to VEGF. Am J Physiol Cell Physiol 2013; 304: C790-C800, doi: 10.1152/ajpcell.00268.2012.

17. Grunewald M, Avraham I, Dor Y, Bachar-Lustig E, Itin A, Jung $S$, et al. VEGF-induced adult neovascularization: recruitment, retention, and role of accessory cells. Cell 2006; 124: 175-189, doi: 10.1016/j.cell.2005.10.036.

18. Zannettino AC, Farrugia AN, Kortesidis A, Manavis J, To LB, Martin SK, et al. Elevated serum levels of stromal-derived factor-1alpha are associated with increased osteoclast activity and osteolytic bone disease in multiple myeloma patients. Cancer Res 2005; 65: 1700-1709, doi: 10.1158/ 0008-5472.CAN-04-1687.

19. Martin SK, Dewar AL, Farrugia AN, Horvath N, Gronthos S, To LB, et al. Tumor angiogenesis is associated with plasma levels of stromal-derived factor-1alpha in patients with multiple myeloma. Clin Cancer Res 2006; 12: 6973-6977, doi: 10.1158/1078-0432.CCR-06-0323.

20. Peled A, Wald O, Burger J. Development of novel CXCR4based therapeutics. Expert Opin Investig Drugs 2012; 21: 341-353, doi: 10.1517/13543784.2012.656197.

21. Katz BZ, Tavor S. The stromal derived factor- $1 \backslash \mathrm{CXCR} 4$ axis - a legitimate therapeutic target in multiple myeloma? Leuk Lymphoma 2009; 50: 1067-1068, doi: 10.1080/104281 90902991837.

22. Mirandola L, Apicella L, Colombo M, Yu Y, Berta DG, Platonova $\mathrm{N}$, et al. Anti-Notch treatment prevents multiple myeloma cells localization to the bone marrow via the chemokine system CXCR4/SDF-1. Leukemia 2013; 27: 1558-1566, doi: 10.1038/leu.2013.27.

23. Beider K, Begin M, Abraham M, Wald H, Weiss ID, Wald O, et al. CXCR4 antagonist 4F-benzoyl-TN14003 inhibits leukemia and multiple myeloma tumor growth. Exp Hematol 2011; 39: 282-292, doi: 10.1016/j.exphem.2010.11.010.

24. Kim SW, Kim HY, Lee HJ, Yun HJ, Kim S, Jo DY. Dexamethasone and hypoxia upregulate CXCR4 expression 
in myeloma cells. Leuk Lymphoma 2009; 50: 1163-1173, doi: 10.1080/10428190902893801.

25. Oliveira AM, Maria DA, Metzger M, Linardi C, Giorgi RR, Moura $\mathrm{F}$, et al. Thalidomide treatment down-regulates SDF-1alpha and CXCR4 expression in multiple myeloma patients. Leuk Res 2009; 33: 970-973, doi: 10.1016/j.leukres.2008.09.018.

26. Keating GM. Plerixafor: a review of its use in stem-cell mobilization in patients with lymphoma or multiple myeloma.
Drugs 2011; 71: 1623-1647, doi: 10.2165/11206040000000000-00000

27. Azab AK, Runnels JM, Pitsillides C, Moreau AS, Azab F, Leleu X, et al. CXCR4 inhibitor AMD3100 disrupts the interaction of multiple myeloma cells with the bone marrow microenvironment and enhances their sensitivity to therapy. Blood 2009; 113: 4341-4351, doi: 10.1182/blood-2008-10186668. 